\title{
Editorial
}

\author{
Luciano da F. Costa \\ Cybernetic Vision Research Group, IFSC, University of São Paulo, \\ Caixa Postal 369, São Carlos, SP 13560-970, Brazil \\ Email:luciano@if.sc.usp.br
}

\section{Fabrice Meriaudeau}

Laboratory Le2i, University of Burgundy, 71200 Le Creusot, France

Email:fabrice@iutlecreusot.u-bourgogne.fr

As a consequence of physical and evolutionary facts, humans became visual creatures to such a degree that the effective execution of most of our activities relies on expert visual abilities. Yet, while humans excel in creative image production (i.e., visual arts) and appreciation, as well as versatile pattern recognition, the use of vision for repetitive inspection purposes is characterized by subjectivity, unreliability, lack of accuracy, and fatigue, and would therefore be better executed by machines. Given the recent advances in both theoretical and practical aspects of signal and imaging sciences, including software and hardware, an exciting technological opportunity is drawn by the possibility of benefiting from the advantages of automation - with its great potential for standardization, dependability, objectivity, precision, and documentability-in the most varied applications.

The objective of the current special issue was exactly to put together a representative collection of articles illustrating the potential of imaging solutions for a large variety of practical applications of automated visual inspection. Acknowledging the pioneering conference series titled Quality Control by Artificial Vision, the current special issue was not only broadly disseminated electronically through Internet, but also among the participants of the 2001 issue of that conference. The high quality of the accepted papers published in this issue provides a clear indication not only that artificial visual inspection has come of age, but also of its remarkable multidisciplinarity and the wide diversity of possible applications.

This special issue starts with four more general and theoretical articles addressing the simulation of specular surface and application to visual inspection, the detection of defects in inclined planes using the Delaunay triangulation, the problem of visual inspection of machined metallic high-precision surfaces, and spatial and spectral approaches to weed detection and localization. The following papers describe the use of imaging concepts and techniques to a whole range of interesting practical problems including wafer defect detection, content-based image retrieval for semiconductor characterization, detection of noncircularity in rolling winders, and quality control for industrial acrylic fiber production. The last two papers address the application of visual inspection to medical/biological problems, namely, quality inspection of mammographic images and multiagent system for edge detection on fish otolith images.

The success of the present special issue would not have been possible without the careful reviewing work undertook by the referees, and the motivation of the authors for addressing and implementing the recommended suggestions and modifications. We are particularly grateful to Nitin Chandrachoodan, for the decisive help with the electronic processing of the submissions, and to Prof. Ray Liu for the constant incentive and effective participation.

Luciano da F. Costa Fabrice Meriaudeau

Luciano da F. Costa was born in São Carlos, São Paulo, Brazil, in 1962. He founded and has coordinated the Cybernetic Vision Research Group (University of São Paulo, São Carlos, Brazil) since 1993. Luciano has got B.Ss. in electronic engineering (University of São Paulo) and computer science (Federal University at São Carlos), an M.S. in applied physics (University of São Paulo), and a Ph.D. in electronics engineering (King's College, University of London). His activity in editorial boards includes EURASIP Journal on Applied Signal Processing (Hindawi), Integrative Neuroscience (World Scientific), 
Journal of Real-Time Imaging (Academic Press), Neuroinformatics (Humana Press), and Psyche (The MIT Press). He has published over 150 refereed articles and is the author (with R. M. Cesar Jr) of the book Shape Analysis and Classification: Theory and Practice, CRC Press, 2001. He is an Associate Professor at the Institute of Physics of São Carlos, University of São Paulo and his research interests include image and shape analysis, pattern recognition, computational biology, and neuro and bioinformatics.

Fabrice Meriaudeau was born in Villeurbanne, France, 1971. He received his engineering degree in material science as well as a Master in physics from the University of Dijon, France, in 1994. He obtained a Ph.D. in physics in May 1997 from the same University. From June 1997 to September 1998, he was a postdoctoral fellow at The Oak Ridge National Laboratory working in the

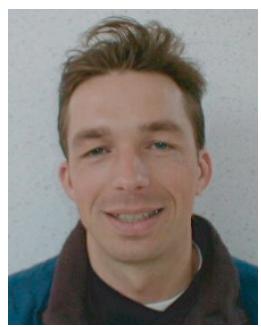
field of biosensing and near field optics. Since September 1998, he has been an Assistant Professor at the Laboratory Le2i of Image Processing in The Institut Universitaire de Technologie (IUT) at Le Creusot, France, where he is engaged in teaching and researching on quality control by artificial vision. He has authored and coauthored more than 80 papers on biosensing and image processing. He was the organizing chairman of the QCAV2001/IEEE conference dedicated to image processing applied to quality control by artificial vision held in Le Creusot in 2001, he is also a member of the scientific committee of SPIE Machine Vision Conference, held every year in San Jose, California. He is acting as a reviewer for various scientific journals: Applied Optics, Optical Engineering. 